\title{
An arithmetization of logical oppositions
}

\author{
Fabien Schang
}

\begin{abstract}
An arithmetic theory of oppositions is devised by comparing expressions, Boolean bitstrings, and integers. This leads to a set of correspondences between three domains of investigation, namely: logic, geometry, and arithmetic. The structural properties of each area are investigated in turn, before justifying the procedure as a whole. Io finish, I show how this helps to improve the logical calculus of oppositions, through the consideration of corresponding operations between integers.
\end{abstract}

Keywords $k$-base system ' Bitstring 'Chasles' relation 'Opposite ' Opposition ' Question-Answer Semantics 'Vectors.

Mathe matics Subject Classification Primary 03B35 · Secondary 03B05 •03B65

\section{Introduction}

The arithmetization of the logic of oppositions it usually taken to mean a process that consists of translating the logical relations between formulas (in a language L) into arithmetic relations between integers (in the domain of positive integers $\mathbf{N}^{*}$ ).

This work can be considered from a structural point of view. That is, just as Descartes made a connection between geometry and arithmetic through analytic geometry, the present paper relies upon the fact that there are blatant analogies between the abstract areas of geometry, logic, and arithmetic. There also exists a form of serendipity in this paper, since the final result is derived from a completely different domain area of discourse - the Chinese Book of Changes (or Yiking). There is, fairly obviously, no causal or logical connection between the latter and logical oppositions, however, a comparison of both domains leads to a fruitful explanation thanks to an analogy between elements of their common structure.

The content of the paper runs as follows. In the first section, we propose a broad historical background of binary systems. In the second section, the geometry of oppositions is considered from the Aristotelian square to recent research developments. In the third section, a logic of opposition is characterized by an algebraic calculus on non-Fregean valuations. In the fourth and final section, we introduce an arithmetic version of oppositions.

\section{A historical background of binary systems}

We start with three main works of Leibniz, the aim of which was (to some extent) to build correspondences between arithmetic and metaphysics: "De progressione Dyadica" (1678), his correspondence (from 1697 to 1703) with Father Joachim Bouvet (1697-1703), a French Jesuit and mathematician sent to China by Louis XIV - above all, his article "Explication de l'arithmétique binaire qui se sert des seuls caractères $0 \& 1$ avec des remarques sur son utilité et sur ce qu'elle donne le sens des anciennes figures chinoises de Fohy" (1703) is a sample of the way in which Leibniz aimed towards his ambitious project of a calculus ratiocinator. Fohy, or $\mathrm{Fu} \mathrm{Xi} \mathrm{(}{ }^{\text {rd }}$ millenary B.C.), is the legendary ancestor of the Chinese who is supposed to have created the well-known Book of Changes, or Yiking. The reason that Leibniz took this religious book into consideration is because of an analogy between the structuration of hexagrams and his own works on the arithmetic binary number system. Furthermore, the 
German philosopher tried to show that a metaphysical interpretation of the Yiking would help to corroborate the Christian metaphysics regarding the origins of Being and Nothingness. We will not discuss this aspect of Leibniz's philosophy, instead, our aim is to show some striking similarities between the Yiking, Leibniz's binary number system, and a Boolean theory of logical oppositions.

Let us consider the binary number system first. Our common number system is decimal, since it consists in ten basic units every numeral is composed of. Leibniz's binary number system relies on the two basic units well-known to computer scientists, viz. the Boolean bits 0 and 1. More generally, there is a systematic way of transcribing in our usual decimal (or 10base) system the number $X$ of an arbitrary number system according to its base and its length. Thus:

For any $k$-base number system in which numbers $(\mathrm{X})_{[k]}$ are sequences of $n$ items, its decimal coding is characterized as follows:

$$
\left(\mathbf{a}_{1} \ldots \mathbf{a}_{n}\right)_{[k]}=\left(\left(k^{n-1} \times \mathbf{a}_{1}\right)+\ldots+\left(k^{0} \times \mathbf{a}_{n}\right)\right)_{[10]}
$$

Let us take an example. What is the appropriate decimal coding of a 2-base number $\mathrm{X}=$ $\left(\mathbf{a}_{1} \ldots \mathbf{a}_{n}\right)_{[2]}$ like, e.g., $(101110)_{[2]}$ ? Its decimal transcription is the integer 46 , or $(46)_{[10]}$, starting from an integer of base $k=2$ and length $n=6$. Indeed,

$$
\begin{aligned}
(101110)_{[2]} & =\left(\left(2^{6-1} \times 1\right)+\left(2^{5-1} \times 0\right)+\left(2^{4-1} \times 1\right)+\left(2^{3-1} \times 1\right)+\left(2^{2-1} \times 1\right)+\left(2^{1-1} \times 0\right)\right)_{[10]} \\
& =\left(\left(2^{5} \times 1\right)+\left(2^{4} \times 0\right)+\left(2^{3} \times 1\right)+\left(2^{2} \times 1\right)+\left(2^{1} \times 1\right)+\left(2^{0} \times 1\right)\right)_{[10]} \\
& =((32 \times 1)+(16 \times 0)+(8 \times 1)+(4 \times 1)+(2 \times 1)+(1 \times 0))_{[10]} \\
& =(32+0+8+4+2+0)_{[10]} \\
& =(46)_{[10]}
\end{aligned}
$$

Here, Leibniz saw a connection between his 2-base number system and the Chinese Yiking, especially the graphic representation of it as set out by the philosopher, numerologist and poet Shao Yong (1012-1077). It is a set of $2^{6}=64$ gua or hexagrams, that is, 6-tuples of lines organized both in a circular and a quadratic form (see Appendix).

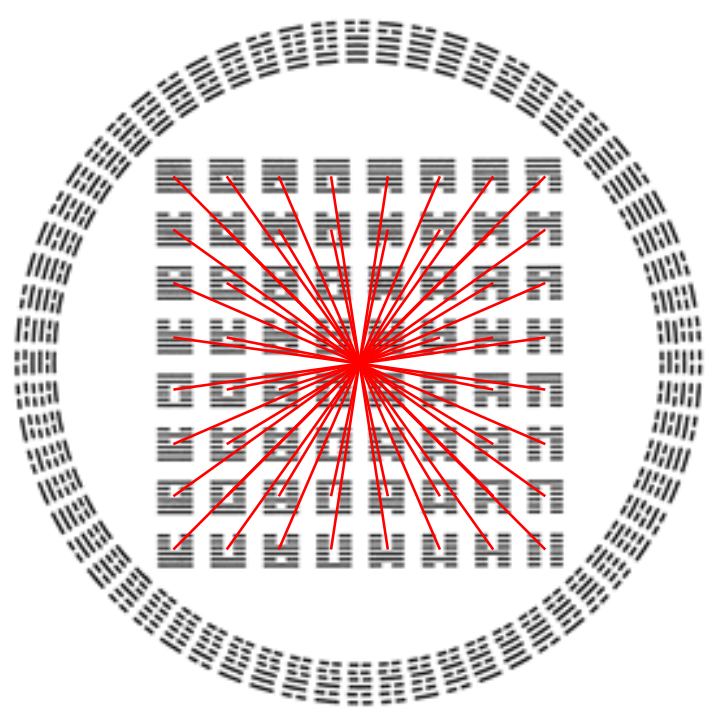


A certain square of contradiction or "earth structure" is included inside the above mandala, or "heaven structure", in the sense that each pair of contradictory terms is similarly related by central symmetry in the square - and it is the same sort of symmetry, which accounts for the distribution of each of the components in the circular mandala. The square and the circle include the same $2^{6}=64$ hexagrams as their components, where 2 is the number $m$ of distinctive data for each item and 6 the number $n$ of items in a given $n$-gram.

Starting from the Daoist picture of the world, each entity of the world is a combination of two basic elements. Subsequently, each discontinuous line of hexagrams symbolizes the passive element Yin, which Leibniz made correspond to the integer 0; each continuous line symbolizes the active element Yang, symbolized by Leibniz with the integer 1. Furthermore, each "gua" (hexagram) historically results from a sequence of two added trigrams, resulting in $2^{3+3}=2^{6}=64$.

However, the Chinese numbering of the hexagrams differs from the Leibnizian representation with respect to its direction (bottom-up for the former, left-right for the latter). For example, the $63^{\text {rd }}$ hexagram is 101010 and has the decimal value $(101010)_{[2]}=(42)_{[10]}$.

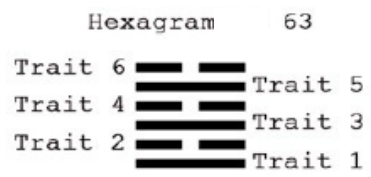

To be read from bottom to top:

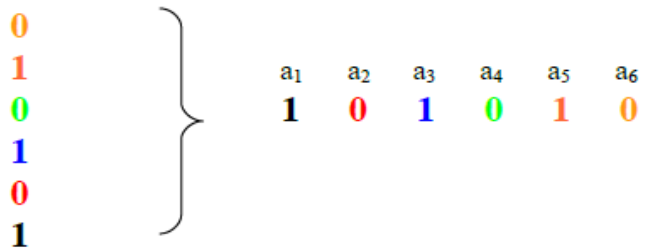

Therefore, the Chinese classification seems to rely on purely contingent rules of interpretation that have nothing to do with arithmetic. So why should we follow Leibniz's binary number system, in this respect?

One reply is to point to the blatant resemblance between the following two examples and recent developments in the theory of opposition.

The first case is the so-called diagram of unity, occurring in the flag of South Korea; it also resorts to a logical octagon of opposition, which is an expansion of the Aristotelian square.
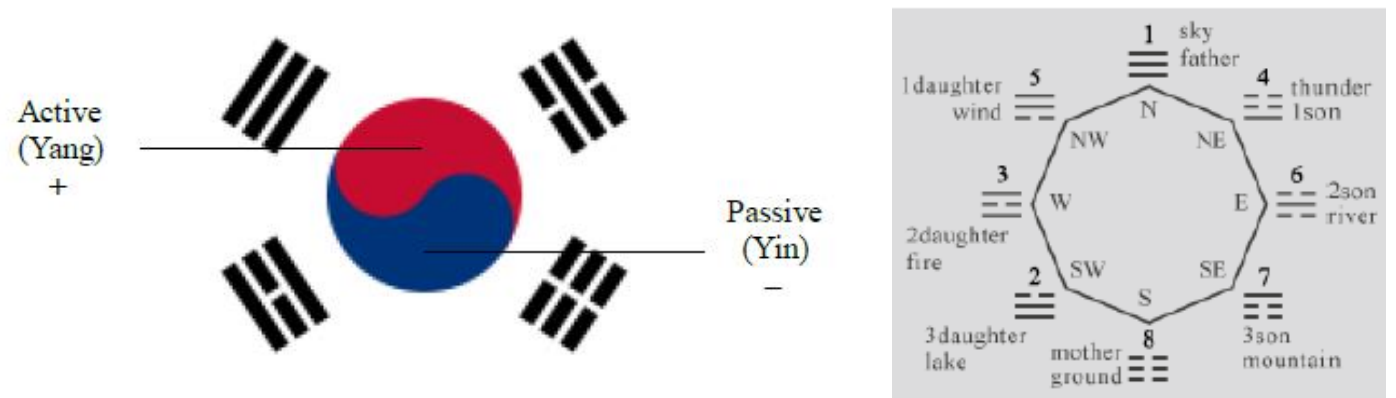

The flag of South Korea stems from four basic elements: Qian (Heaven), Kan (Water), Kun (Earth), and $\mathrm{Li}$ (Fire). However, its four components result from a combination of three lines 
whose exhaustive set is depicted clockwise in the middle figure. By adapting the eight trigrams of the preceding right hand figure to the Leibnizian 2-base number system, we obtain these definitions: Thunder (first son): $(001)_{[2]}=1$; River (second son): $(010)_{[2]}=2$; Mountain (third son): $(100)_{[2]}=4$; Ground (mother): $(000)_{[2]}=0$; Lake (third daughter): $(011)_{[2]}=3$; Fire (second daughter): $(101)_{[2]}=5$; Wind (first daughter): $(110)[2]_{[2]}=6$; Sky (father): $(111)_{[2]}=$ 7. It is important to note that the boldface numbers placed above the trigrams do not correspond to their decimal translation: they represent ordinal numbers ( $\mathbf{1}$ for 'the $1^{\text {st }}$, trigram", 2 for the $2^{\text {nd }}$ trigram, and so on), just as in the various sequences proposed throughout the history of the Yiking (Shao Yong, Jin Fang, Mawangdui).

The same distribution of relations occurs in the below logical octagon below, which is a double extension of the initial square of oppositions: two additional pairs of edges supplement the hexagon vertically $-\mathbf{U}$ and $\mathbf{Y}$, and horizontally $-\ddot{\mathbf{U}}$ and $\ddot{\mathbf{Y}}$. The resulting octagon is a combination of the diagrams studied in [2-4]: the two hexagons AUEOYI and AE YOOIÜ are combined to form the octagon AUEŸOYIÜ, which was also mentioned in [1] for studies on modal logic.

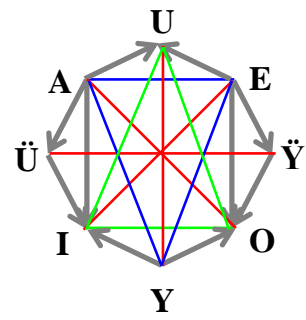

The isomorphism between the Yiking trigram and logical octagons does not mean that they match with each other, however: each vertex of the logical octagon corresponds to a bitstring of length $n=4$, whereas trigrams are bitstrings of length $n=3$. This is the case, because the first and eighth trigrams (Sky and Ground, respectively) have the maximal and minimal values 8 and 0 ; these extrema correspond to tautology and antilogy in logical polygons, and both can be located at the center of intersection of all the logical contradictories (see page 7).

The second case is the Seal of Solomon, also occurring in a flag -that of Israel. Whilst the Korean flag has just been compared to the logical octagon, the present one also corresponds to one diagram of logical oppositions: Blanché's logical hexagon of oppositions AUEOYI, once the latter has been deprived from its surrounding relations of subalternation.
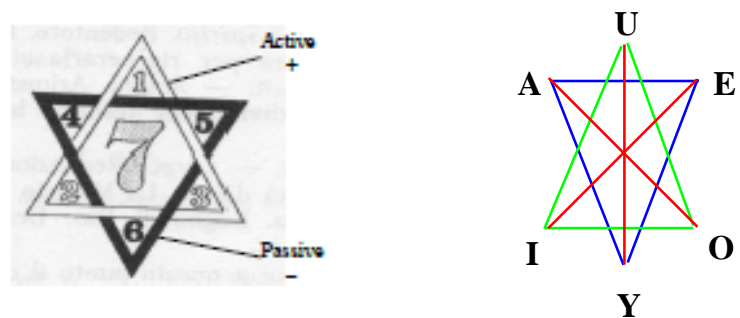

This truncated version of the hexagram is a six-point starlike figure of the the Magen David (Shield of David), to be also compared with the Hindu Shatkona. Each edge of the Seal is marked with an integer from 1 to 6: 4 for $\mathbf{A}, 1$ for $\mathbf{U}, 5$ for $\mathbf{E}, 3$ for $\mathbf{O}, 6$ for $\mathbf{Y}, 2$ for $\mathbf{I}$. The center of the star is marked with the integer 7, resulting from the sum of each of its diagonals: $(1+6)=(2+5)=(3+4)=7$.

The connection link between this mystic star and the logical hexagon is not obvious at first sight, given that the opposition between numbers and sentences is not supposed to obey one and the same ordering process. Yet such a connection is made possible through arithmetic, 
especially Leibniz's 2-base system, as will be shown in the section devoted to the logic of opposition.

It should be taken for granted that the above two examples of the Yiking and the Seal of Solomon do not constitute a plea for numerology. The present paper does not purport to show the "power" of numbers in order to explain how things are organized in the world, as is the case with the 64 exhaustive elements of the world in the Yiking. Rather, this paper is an essay on numerical logic and deals with the "power" of numbers in order to show how people think logically. It is a matter of hermeneutics, i.e., how to interpret signs in a language, whether signs denote integers in number theory or propositions in logic.

To investigate this, three languages are compared in the next sections: geometrical, logical, and arithmetic languages, assuming that each of the three areas consists of a set of structured elements with specific relations between them.

\section{Geometry of oppositions}

The most famous geometry of oppositions is the so-called Aristotelian square, originating from the logical corpus of the Stagirite. Although the latter did not himself consider the logical oppositions of contradiction and contrariety in this way, the "Aristotelian" square refers to the set of 6 logical oppositions between the categorical propositions A,E,I,O of syllogistics. Despite some isolated developments of the geometry of oppositions in the history of logic $-e . g$., Buridan's logical octagon of de re modalities, a new impetus has been given much later by the French philosopher and logician Robert Blanché. As exemplified above, Blanché's hexagon has enriched the Aristotelian square with two additional vertices $\mathbf{Y}, \mathbf{U}$ in the vertical sense and given several interpretations to the six resulting vertices: quantified, modal, but also conceptual in a broader way. Earlier, the Polish logician Czeżowski extended Aristotle's categorical square by introducing the singular propositions $\ddot{\mathbf{Y}}, \ddot{\mathbf{U}}$. This suggests that there was no transcendental limitation on the initial square of oppositions, insofar as more or less than four propositions may be opposed to each other. Moreover, propositions are not the only sort of meaningful entities that can be represented in such geometrical figures: concepts, modalities, and so on, may also be defined in terms of opposition.

A more systematic treatment of logical oppositions has been recently proposed by philosophers, linguists, and mathematicians [1-5-6-10]. Some precise explanations have been given there about the minimal and maximal extensions of the historical core pattern of geometrical oppositions, namely: the square. There are not only different extensions of the opposed edges in an arbitrary figure, but also different ways of representing these extensions according to their inner structure.

Even given this precedence, there may remain some reluctance towards such a logical procedure: why and how to afford a geometrical representation for logical oppositions? But, whilst the core logical notion of consequence seems absent from this special discipline, we see at least two advantages of a geometry of oppositions.

On the one hand, these structured geometries offer a harmonious representation of logical contradictions by central symmetry. This has been also seen twice above, both with logical hexagons and octagons as well as in the mystic figures of the Yiking and the Seal of Solomon. The central symmetry is shown there by sets of intersecting lines between red contradictories: two lines in a square, three lines in a hexagon, four lines in a octagon, and so on, until a circle displaying a hypothetically complete set of $n$ relations in a perfect shape (recall Shao Yong's aforementioned mandala). 


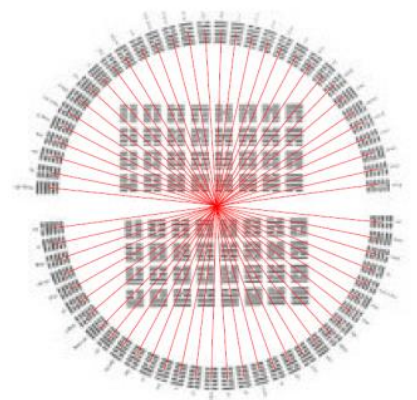

This suggests that such a way of organizing oppositions goes beyond the scientific domain of modern logic and already occurred a long time ago in prescientific domains of thought.

On the other hand, a geometrical figure may also be used to depict the structural completeness of a finite set of logical relations. To the question regarding how many logical relations there can be in a given set of propositions or concepts, the answer is that this depends on the number of relata to be opposed to each other in a given geometrical structure. Thus, a logical structure (square, hexagon, and the like) is said to be complete if and only if al the logical relations can be displayed in it. Following [8-11], structural completeness can be explained as follows:

For any oppositional structure with $m$ relata, the number of $n$-ary relations between any relata is a combination $\mathrm{C}_{m}{ }^{n}=m ! /(n !(m-n) !)$.

For example, the Aristotelian square of oppositions is a structure that includes $m=4$ relata; hence there is a set of $\mathrm{C}_{4}{ }^{2}=4 ! /(2 !(4-2) !)=6$ binary relations between its relata: AE,AO,AI,EO,EI,OI.

A related problem was raised in [2]: how to display every such opposition between the classical (bivalent) binary sentences in one and the same geometric structure?

Binary sentences are of the form $p \circ q$, where $\circ$ is a binary connective. There is a total set of $2^{n m}=2^{22}=2^{4}=16$ such connectives in the classical or two-valued logic, where $n$ stands for number of truth-values ( $\mathrm{T}$ for truth, $\mathrm{F}$ for falsehood) and $m$ for the number of connected propositions $(p$ and $q)$.

A partial solution has been proposed by [2] in the form of hexadic structure. It is a repeated two-by-two connection of hexagons, each being related by two common vertices up and down and forming a whole DNA-shaped structure of ten elements (a)-(j).

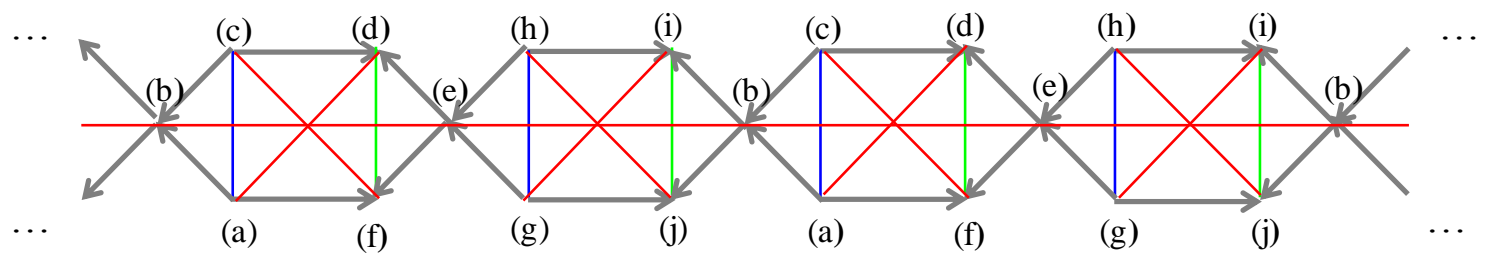

(a) $p \wedge q$; (b) $p \leftrightarrow q$; (c) $\sim(p \vee q)$; (d) $\sim(p \wedge q)$; (e) $\sim(p \leftrightarrow q)$; (f) $p \vee q$; (g) $\sim(p \leftarrow q)$; (h) $\sim(p \rightarrow q)$; (i) $p \leftarrow q ;(\mathrm{j}) p \rightarrow \mathrm{q}$

Albeit an extension of the single hexagon, the above structure is still incomplete since 6 among the 16 binary connectives are still missing there.

A solution has been found by [6] in order to introduce all binary connectives into one and the same structure while following the basic criterion of central symmetry for contradictories. The result is a tetraicosahedron of logical oppositions, central symmetry requiring a transition from $2 \mathrm{D}$ to $3 \mathrm{D}$ geometry. It is a very complex figure of $\mathrm{C}_{16^{2}}{ }^{2}=16 ! /(2 !(16-2) !)=120$ binary 
relations including 8 contradictories, in which the special connectives of tautology and antilogy are located in the center of the structure. A common feature with the Seal of Solomon is the occurrence of special values in the center of the figure: tautology and antilogy, while the Seal has the sum of any opposed integers as its core value. The link between the latter degenerate connectives $T, \perp$ and the arithmetic notion of sum will be explained in the final section.

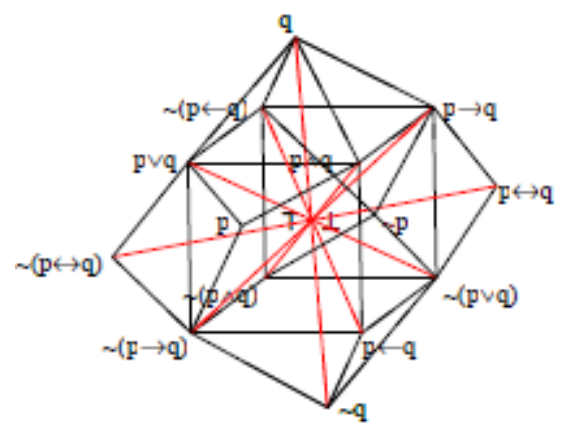

The above geometric structure [5,6] includes 6 squared faces; however, these are not Aristotelian squares because they fail to have some of the usual logical relations in them in such a $3 \mathrm{D}$ structure. The latter also includes 10 hexagons, whether regular or irregular. Some of these figures have been studied at length elsewhere, especially in [5-10], and it is not the purpose of the present paper to scrutinize the geometric features of logical oppositions. Rather, our point is to show how the link can be made between geometry and arithmetic through logic and with respect to the same issue: opposition.

\section{Logic of oppositions}

The following logic of oppositions departs from mainstream systems by focusing on the concept of opposition instead of consequence. It is not a logical system endowed with a set of theorems; rather, its purpose is to give an abstract formal definition of logical relations including cases of opposition.

It is well-known that the truth-functional classical or two-valued logic is not able to characterize each of the logical relations of opposition: if any two sentences $\varphi_{1}, \varphi_{2}$ are said to be contraries (or subcontraries) what of the truth-value of $\varphi_{2}$ if $\varphi_{1}$ is false (or true)? The impossibility to treat oppositions as functions thus necessitates another formal device to deal with logical oppositions.

\subsection{Opposition and opposites}

This has been developed in several respects [7-8-9], inspired by two previous formal devices: $\mathrm{P}^{\mathrm{Q}}$-semantics, introduced in [5]; Modal Quantified Algebra, elaborated in Smessaert [10]. Let us consider the resulting general theory of oppositions. It consists of two main sections, namely, a semantic of the relations of opposition, and a complementary theory of opposites, which are relata formed by opposition-forming operators.

The basic semantics embracing both areas is a constructive Question-Answer Semantics (hereafter, QAS). It is an algebraic and non-Fregean semantics: in a Fregean semantics, logical values are "truth-values" (true, or false) corresponding to single non-structured objects and are assigned to only one category of objects, viz. propositions. In QAS, logical values are structured context-dependent objects and are assigned to any meaningful expression (not only sentences, but also concepts or terms). In the case of sentential expressions, the meaning of any sentence is afforded by a finite sequence of answers to corresponding questions about the 
sentence. It results in an alternative coding of the logical values of any sentence $\varphi_{i}$ : not $\mathrm{T}$ or $\mathrm{F}$, but a bitstring of relative length. The process of valuation can be defined generally as follows:

For any meaningful expression $\varphi$, there are $m^{n}$ logical values in a semantic with:

- $n$ questions $\mathbf{Q}(\varphi)=\left\langle\mathbf{q}_{1}(\varphi), \ldots, \mathbf{q}_{\mathrm{n}}(\varphi)\right\rangle$

- $m$ sorts of answer $\mathbf{A}(\varphi)=\left\langle\mathbf{a}_{1}(\varphi), \ldots, \mathbf{a}_{\mathrm{n}}(\varphi)\right\rangle$, every element $\mathbf{a}(\varphi)$ mapping into $\{m-1, \ldots, 0\}$.

The presentation of logical relations in the form of a question-answer game helps to approach an important problem concerning their cardinality, namely: how many logical oppositions can there be? Although it is usually said that the Aristotelian square is a set of four "oppositions", a more scrutinized investigation will show that this is not so.

In the following logic of oppositions, only $m=2$ sorts of answer are used as single bits: yes (symbol: 1), and no (symbol: 0). A corresponding semantics of classical oppositions consists in interpreting binary sentences in the form of sequences of 4-bits, each logical value standing for their Disjunctive Normal Form. The sense $\mathbf{Q}(\varphi)=\left\langle\mathbf{q}_{1}(\varphi), \mathbf{q}_{2}(\varphi), \mathbf{q}_{3}(\varphi), \mathbf{q}_{4}(\varphi)\right\rangle$ of these binary sentences $\varphi=p \circ q$ is specified by an ordered set of 4 questions about their possible valuations: $\mathbf{q}_{1}(\varphi)$ : “ $v(p)=v(q)=\mathrm{T}$ ?"; $\mathbf{q}_{2}(\varphi)$ : “ $v(p)=\mathrm{T}, v(q)=\mathrm{F}$ ?"; $\mathbf{q}_{3}(\varphi)$ : “ $v(p)=\mathrm{F}$, $v(q)=\mathrm{T}$ ?"; $\mathbf{q}_{4}(\varphi)$ : “ $v(p)=v(q)=\mathrm{F}$ ?".

This results in a set of $2^{4}=16$ logical values $\mathbf{A}(\varphi)=\left\langle\mathbf{a}_{1}(\varphi), \mathbf{a}_{2}(\varphi), \mathbf{a}_{3}(\varphi), \mathbf{a}_{4}(\varphi)\right\rangle$, each of these characterizing one of the 16 binary sentences of two-valued logic.

$\begin{array}{llll}p \circ q=\varphi & \mathbf{A}(p \circ q)=\mathbf{A}(\varphi) & p \circ q=\varphi & \mathbf{A}(p \circ q)=\mathbf{A}(\varphi) \\ p \vee \sim p & 1111=\mathrm{T} & p \leftrightarrow q & 1001 \\ p \vee q & 1110 & q & 1010 \\ p \longleftarrow q & 1101 & \sim q & 0101 \\ p \rightarrow q & 1011 & \sim(p \vee q) & 0001 \\ \sim(p \wedge q) & 0111 & \sim(p \leftarrow q) & 0010 \\ p & 1100 & \sim(p \rightarrow q) & 0100 \\ \sim(p \leftrightarrow q) & 0110 & p \wedge q & 1000 \\ \sim p & 0011 & \perp=p \wedge \sim p & 0000\end{array}$

Once the logical values are set out, it becomes possible to achieve a calculus of oppositions and opposites [7]. All related concepts are characterized by two interconnected logical forms. On the one hand, oppositions are relations the form $\operatorname{Op}\left(\varphi_{1}, \varphi_{2}\right)$ and can be read as " $\varphi_{1}$ stands in an opposition of ... to $\varphi_{2}$ ". The generic relation $\mathrm{R}=\mathrm{Op}$ is depicted in all geometric structures we dealt with previously, Aristotle's square, Blanché's hexagon, and so on, with the relata $\varphi_{1}, \varphi_{2}$, and the relation $\mathrm{Op}$, corresponding to vertices and lines.

On the other hand, opposites are opposition-forming operators mapping on expressions and forming non-identical expressions in a given set of logical values. The generic oppositionforming operator is of the form $\operatorname{op}\left(\varphi_{1}\right)=\varphi_{2}$. This includes its output value and can be read as "a ... of $\varphi_{1}$ is $\varphi_{2}$ ", so that any application of an operator op yields a corresponding relation from the set $\mathrm{Op}=\{\mathrm{CT}, \mathrm{CD}, \mathrm{SCT}, \mathrm{NCD}\}$.

Let $\Pi$ and $\sqcup$ be the Boolean operations of meet and join such that, for any single answer $\mathbf{a}_{\mathrm{i}}(\varphi)$ :

$\mathbf{a}_{\mathrm{i}}\left(\varphi_{1}\right) \sqcap \mathbf{a}_{\mathrm{i}}\left(\varphi_{2}\right)=\min \left(\mathbf{a}_{\mathrm{i}}\left(\varphi_{1}\right), \mathbf{a}_{\mathrm{i}}\left(\varphi_{2}\right)\right)$

$\mathbf{a}_{\mathrm{i}}\left(\varphi_{1}\right) \sqcup \mathbf{a}_{\mathrm{i}}\left(\varphi_{2}\right)=\max \left(\mathbf{a}_{\mathrm{i}}\left(\varphi_{1}\right), \mathbf{a}_{\mathrm{i}}\left(\varphi_{2}\right)\right)$ 
Then for every relation $\operatorname{Op}\left(\mathbf{A}\left(\varphi_{1}\right), \mathbf{A}\left(\varphi_{2}\right)\right)=\operatorname{Op}\left(\mathbf{A}\left(\varphi_{1}\right), \mathbf{A}\left(\operatorname{op}\left(\varphi_{1}\right)\right)\right)$, we have the following distinctive valuations:

Contrariety: CT

$\operatorname{Op}\left(\varphi_{1}, \varphi_{2}\right)=\operatorname{CT}\left(\varphi_{1}, \operatorname{ct}\left(\varphi_{1}\right)\right)$ iff

$\mathbf{A}\left(\varphi_{1}\right) \sqcap \mathbf{A}\left(\varphi_{2}\right)=\perp$ and $\mathbf{A}\left(\varphi_{1}\right) \sqcup \mathbf{A}\left(\varphi_{2}\right) \neq \mathbf{T}$

Contradictoriness: $\mathrm{CD}$

$\operatorname{Op}\left(\varphi_{1}, \varphi_{2}\right)=\operatorname{CD}\left(\varphi_{1}, \operatorname{cd}\left(\varphi_{1}\right)\right)$ iff

$\mathbf{A}\left(\varphi_{1}\right) \sqcap \mathbf{A}\left(\varphi_{2}\right)=\perp$ and $\mathbf{A}\left(\varphi_{1}\right) \sqcup \mathbf{A}\left(\varphi_{2}\right)=\mathrm{T}$

Subcontrariety: SCT

$\operatorname{Op}\left(\varphi_{1}, \varphi_{2}\right)=\operatorname{SCT}\left(\varphi_{1}, \operatorname{sct}\left(\varphi_{1}\right)\right)$ iff

$\mathbf{A}\left(\varphi_{1}\right) \sqcap \mathbf{A}\left(\varphi_{2}\right) \neq \perp$ and $\mathbf{A}\left(\varphi_{1}\right) \sqcup \mathbf{A}\left(\varphi_{2}\right)=\mathrm{T}$

Non-Contradictoriness: NCD

$\operatorname{Op}\left(\varphi_{1}, \varphi_{2}\right)=\operatorname{NCD}\left(\varphi_{1}, \operatorname{ncd}\left(\varphi_{1}\right)\right)$ iff

$\mathbf{A}\left(\varphi_{1}\right) \sqcap \mathbf{A}\left(\varphi_{2}\right) \neq \perp$ and $\mathbf{A}\left(\varphi_{1}\right) \sqcup \mathbf{A}\left(\varphi_{2}\right) \neq \mathbf{T}$

It is worthwhile to note that the well-known relation of subalternation SB does not appear in the above list: its valuation cannot be made distinct from non-contradictoriness, and another way to individuate SB goes by the following definition:

Subalternation: SB

$\operatorname{Op}\left(\varphi_{1}, \varphi_{2}\right)=\operatorname{SB}\left(\varphi_{1}, \operatorname{sb}\left(\varphi_{1}\right)\right)$ iff

$\mathbf{A}\left(\varphi_{1}\right) \sqcap \mathbf{A}\left(\varphi_{2}\right)=\mathbf{A}\left(\varphi_{1}\right)$ and $\mathbf{A}\left(\varphi_{1}\right) \sqcap \mathbf{A}\left(\varphi_{2}\right) \neq \mathbf{A}\left(\varphi_{2}\right)$

Subalternation has two peculiar features. For one thing, it has a converse relation of superalternation $\mathrm{SB}^{-1}=\mathrm{SP}$, by reverting the relation order between its relata $\varphi_{1}$ and $\varphi_{2}$. Also, it is not characterized in the same way as the previous relations: these are defined by a combinatorial game of minimal and maximal valuations, $\min \left(\varphi_{i}\right)=\perp$ and $\max \left(\varphi_{i}\right)=\mathrm{T}$, depending upon whether their meet and join values result or not in either of these extreme valuations. There is still one such combination that has never been introduced in the usual theory of opposition: unconnectedness [10-11], which imposes absolutely no constraint on the relata. However, this further relation cannot be defined by the sole terms of opposition and requires another sort of questioning: not only about difference, but also about identity.

\subsection{Identities and differences}

The above definitions show that the concept of subalternation does not answer to the same set of questions as the other four relations. Following [11], one can see the whole as a set of two distinctive questionings about modes of difference and identity.

On the one side, the sense of logical oppositions is given by two meta-questions about differences in valuations: 
$\mathbf{Q}\left(\mathrm{Op}\left(\varphi_{1}, \varphi_{2}\right)\right)=\left\langle\mathbf{q}_{1}\left(\mathrm{Op}\left(\varphi_{1}, \varphi_{2}\right)\right), \mathbf{q}_{2}\left(\mathrm{Op}\left(\varphi_{1}, \varphi_{2}\right)\right)\right\rangle$

$\mathbf{q}_{1}\left(\mathrm{Op}\left(\varphi_{1}, \varphi_{2}\right)\right)$ : “ $\mathbf{a}_{i}\left(\varphi_{1}\right)=0 \Rightarrow \mathbf{a}_{i}\left(\varphi_{2}\right) 1$ ?"

$\mathbf{q}_{2}\left(\mathrm{Op}\left(\varphi_{1}, \varphi_{2}\right)\right)$ : “ $\mathbf{a}_{i}\left(\varphi_{1}\right)=1 \Rightarrow \mathbf{a}_{i}\left(\varphi_{2}\right) 0$ ?"

Contradiction, contrariety, subcontrariety and unconnectedness are rendered by these questions: $\mathbf{A}\left(\operatorname{CD}\left(\varphi_{1}, \varphi_{2}\right)\right)=\langle 1,1\rangle, \quad \mathbf{A}\left(\operatorname{CT}\left(\varphi_{1}, \varphi_{2}\right)\right)=\langle 0,1\rangle, \quad \mathbf{A}\left(\operatorname{SCT}\left(\varphi_{1}, \varphi_{2}\right)\right)=\langle 1,0\rangle$, and $\mathbf{A}\left(\operatorname{NCD}\left(\varphi_{1}, \varphi_{2}\right)\right)=\langle 0,0\rangle$.

On the other side, the sense of logical implications is given by two meta-questions about identities in valuation:

$\mathbf{Q}\left(\operatorname{Imp}\left(\varphi_{1}, \varphi_{2}\right)\right)=\left\langle\mathbf{q}_{1}\left(\operatorname{Imp}\left(\varphi_{1}, \varphi_{2}\right)\right), \mathbf{q}_{2}\left(\operatorname{Imp}\left(\varphi_{1}, \varphi_{2}\right)\right)\right\rangle$

$\mathbf{q}_{1}\left(\operatorname{Imp}\left(\varphi_{1}, \varphi_{2}\right)\right)$ : “ $\mathbf{a}_{i}\left(\varphi_{1}\right)=1 \Rightarrow \mathbf{a}_{i}\left(\varphi_{2}\right) 1$ ?"

$\mathbf{q}_{2}\left(\operatorname{Imp}\left(\varphi_{1}, \varphi_{2}\right)\right):$ : $\mathbf{a}_{\mathrm{i}}\left(\varphi_{1}\right)=0 \Rightarrow \mathbf{a}_{i}\left(\varphi_{2}\right) 0$ ?"

Subalternation is rendered transparent by this set of questionings, highlighting its peculiarity with respect to the preceding relations of opposition: $\mathbf{A}\left(\operatorname{SB}\left(\varphi_{1}, \varphi_{2}\right)\right)=\langle 1,0\rangle$. Special headings are assigned in [11] to the other three identity relations: bi-implication for $\mathbf{A}\left(\operatorname{Imp}\left(\varphi_{1}, \varphi_{2}\right)\right)=$ $\langle 1,1\rangle$, right implication for $\mathbf{A}\left(\operatorname{Imp}\left(\varphi_{1}, \varphi_{2}\right)\right)=\langle 0,1\rangle$, and non-implication for $\mathbf{A}\left(\operatorname{Imp}\left(\varphi_{1}, \varphi_{2}\right)\right)=$ $\langle 0,0\rangle$.

On the one hand, it quickly appears that the 16 binary connectives result from a combination of the above four questionings, thus accounting for their cardinality. However, the questioning giving rise to the sixteen Disjunctive Normal Forms includes notions of compossibility and asks if any two sentences can be true or false together or not. At the same time, the meta-questioning that has just been used for relations of difference and opposition deals with necessary relations, having to do with metalogical possibilities and necessities.

On the other hand, every logical relation results from a combination of identities and differences, viz. between the bits of a given expression and those of its relatum. In this sense, it can be said that every logical relation is a logical opposition, once opposition is defined in QAS as a Boolean difference of relative degree between bitstrings. It has been argued in [11] that subalternation is not a relation of opposition at all, as depicted by the above distinction between questionings about differences and identities (subalternation proceeds from the latter). Admittedly, no logical relation is to be properly called an "opposition" if its relata are compatible with each other. But since this also holds for subcontrariety and unconnectedness, our reply is that a calculus of opposites helps to show that any different relata can be formed by means of contradictory oppositions between some of their single bits. Thus, a constructive definition of relations entails that different relata may be opposed in some way to each other, whilst being possibly compatible with respect to their whole bitstrings.

\subsection{Negations}

It has been argued in [7] that each of these opposition-forming operators is a special case of negation: a difference-forming operator that turns a given value into another one. However, these operators are not functions in the usual sense of the word. That is to say, most of these are not one-to-one mappings turning one input value into another single one. Rather, the opposition-forming operators op are mostly one-many mappings: to one input value corresponds more than one output value.

On the one hand, contradiction is the only extensional opposition-forming operator. In other words, the operator $\mathrm{op}_{x}=\mathrm{cd}$ is a bijection. This is shown arithmetically by the fact that there 
is only one integer $\mathbf{A}\left(\varphi_{1}\right)+\mathbf{A}\left(\varphi_{2}\right)=\max \left(\varphi_{i}\right)$. By derivation, only classical negation plays the role of a contradiction-forming operator.

On the other hand, contrariety-forming operators can be associated to what is called a paracomplete negation, whilst subcontrariety-forming operators behave like paraconsistent negation. Such a correspondence relies upon the nature of consequence from the perspective of logical oppositions. As far as one can see, subalternation is the best candidate to render the notion of consequence in a Boolean version of logical oppositions. Indeed, every premise is such that it entails a number of consequences, and this number is dependent upon the ways every yes-answer of the premise can be contained within them. Taking the case of conjunction, $p \wedge q$, Boolean oppositions combined with an informal definition of subalternation helps to show that binary conjunction has as many consequences as logical contraries. According to [1], subaltern expressions correspond to contradictories of contraries.

It has also been shown in $[8,11]$ that the number of subalterns and contrary is relative to the bitstring $\mathbf{A}\left(\varphi_{i}\right)$ characterizing any expression $\varphi_{i}$. Thus, not every expression has contraries or subcontraries, by virtue of its Boolean value, whereas every expression has its own contradictory. In this way, we get information both about the number of logical opposites and the nature of any opposition between arbitrary relata.

Moreover, a general calculus of oppositions can be achieved through two basic operators, cd and $\mathrm{ct}$, in addition to the method of substitution. Then, for every expression $\varphi_{i}$ :

$$
\begin{aligned}
& \operatorname{sb}\left(\varphi_{i}\right)=\operatorname{cd}\left(\operatorname{ct}\left(\varphi_{i}\right)\right) \\
& \operatorname{sct}\left(\varphi_{i}\right)=\operatorname{cd}\left(\operatorname{sp}\left(\varphi_{i}\right)\right) \\
& \operatorname{sp}\left(\varphi_{\mathrm{i}}\right)=\operatorname{ct}\left(\operatorname{cd}\left(\varphi_{\mathrm{i}}\right)\right)
\end{aligned}
$$

However, the behavior of op as a one-many operator is troublesome from a computational point of view: how to determine the value of, for example, the contrary of a contradictory expression, if there may be more than one output value? The next section helps to clarify these issues.

\section{Arithmetic of oppositions}

In the preceding algebraic logic of oppositions, the logical import of relations relies upon a Boolean calculus of expressions. Again, in the general logical form

$$
\begin{gathered}
\Sigma(\varphi)_{[k]}=\sigma_{1}(\varphi)_{[k]}+\ldots+\sigma_{\mathrm{n}}(\varphi)_{[k]}, \\
\quad \text { where } \sigma_{i}(\varphi)_{[k]}=\left(k_{i-1}=\mathbf{a}_{i}(\varphi)\right)
\end{gathered}
$$

Arithmetic counterparts of the logical relation Op, and its corresponding logical operators op, are the relation of difference $\oplus$ and its corresponding operators of differentiation \pm in $\mathbf{N}^{*}$. In addition, just as we expressed the view that oppositions are formed by means of oppositionforming operators, we also can say that, for any pair of integers $\Sigma\left(\varphi_{i}\right)_{[k]}, \Sigma\left(\varphi_{j}\right)_{[k]}$ :

$$
\oplus\left(\Sigma\left(\varphi_{i}\right)_{[k]}, \Sigma\left(\varphi_{j}\right)_{[k]}\right)=\oplus\left(\Sigma\left(\varphi_{i}\right)_{[k]}, \pm\left(\Sigma\left(\varphi_{i}\right)_{[k]}\right)\right) .
$$

In other words, the relation $\oplus\left(\Sigma\left(\varphi_{i}\right)_{[k]}, \Sigma\left(\varphi_{j}\right)_{[k]}\right)$ stands for an arithmetic difference expressed by an integer of $\mathbf{N}^{*}$ such that $\left.\oplus\left(\Sigma\left(\varphi_{i}\right)[k]\right) \neq \Sigma\left(\varphi_{j}\right)_{[k]}\right)$. It corresponds to Leibniz's 2-base number and its decimal coding, such that

$$
\oplus\left(\Sigma\left(\varphi_{i}\right)_{[2]}, \Sigma\left(\varphi_{j}\right)_{[2]}\right)=2^{n-1} \times\left(\mathbf{a}_{1}\left(\varphi_{i}\right)-\mathbf{a}_{1}\left(\varphi_{j}\right)\right)+\ldots+2^{0} \times\left(\mathbf{a}_{n}\left(\varphi_{i}\right)-\mathbf{a}_{n}\left(\varphi_{j}\right)\right)
$$


The set of binary sentences and their arithmetic value can be listed as follows.

$\begin{array}{llllll}p \circ q=\varphi & \mathbf{A}(p \circ q) & \Sigma(p \circ q) & p \circ q=\varphi & \mathbf{A}(p \circ q) & \Sigma(p \circ q) \\ p \vee \sim p & 1111 & 15 & p \leftrightarrow q & 1001 & 9 \\ p \vee q & 1110 & 14 & q & 1010 & 10 \\ p \leftarrow q & 1101 & 13 & \sim q & 0101 & 5 \\ p \rightarrow q & 1011 & 11 & \sim(p \vee q) & 0001 & 1 \\ \sim(p \wedge q) & 0111 & 7 & \sim(p \leftarrow q) & 0010 & 2 \\ p & 1100 & 12 & \sim(p \rightarrow q) & 0100 & 4 \\ \sim(p \leftrightarrow q) & 0110 & 6 & p \wedge q & 1000 & 8 \\ \sim p & 0011 & 3 & p \wedge \sim p & 0000 & 0\end{array}$

For example, let $\varphi_{1}=p \wedge q, \varphi_{2}=p \vee q$. On the one hand, binary sentences $\varphi_{1}$ and $\varphi_{2}$ are related by a relation of subalternation $\operatorname{Op}\left(\mathbf{A}\left(\varphi_{1}\right), \mathbf{A}\left(\varphi_{2}\right)\right)=\operatorname{SB}(1000,1110)$. On the other hand, the operation turning the arithmetic value of the first relatum into the second one is -6 :

$$
\begin{aligned}
\oplus\left(\Sigma\left(\varphi_{1}\right)_{[2]}, \Sigma\left(\varphi_{2}\right)_{[2]}\right) & =\left(2^{4-1} \times(1-1)\right)+\left(2^{3-1} \times(0-1)\right)+\left(2^{2-1} \times(0-1)\right)+\left(2^{1-1} \times(0-0)\right) \\
& =(8 \times 0)+(4 \times(-1))+(2 \times(-1))+(1 \times 0) \\
& =0+(-4)+(-2)+0 \\
& =-6
\end{aligned}
$$

The second step of our analogy consists in giving an arithmetic sense to the logical concept of opposition.

\subsection{Arithmetical oppositions}

It may seem impossible to talk about oppositions between integers. Although I have already shown that concepts can be opposed to each other without referring to truth-values, it hardly makes sense to say that, e.g., 7 is opposed to 5 by some relation of opposition. For this purpose, an additional arithmetic criterion is required, namely, the introduction of maximal of minimal integers, just as tautologies and antilogies play this role of extreme values in algebraic logic. Thus for every sequence of $n$ items $\varphi_{i}$ in a $k$-base system:

- its maximum or maximal value is $\Sigma\left(\varphi_{i}\right)=k^{n-1}$

Example: if $k=2$ and $n=4$, then $\max \left(\Sigma\left(\varphi_{i}\right)\right)=2^{4-1}=15$

- its minimum or minimal value is $\Sigma\left(\varphi_{i}\right)=0$

Maximal value helps to understand why every vertex has only one contradictory: there is only one $\Sigma\left(\varphi_{2}\right)$ such that $\Sigma\left(\varphi_{1}\right)+\Sigma\left(\varphi_{2}\right)=\max \left(\Sigma\left(\varphi_{i}\right)\right)$, and $\operatorname{Op}\left(\Sigma\left(\varphi_{1}\right), \Sigma\left(\varphi_{2}\right)\right)=\operatorname{CD}\left(\Sigma\left(\varphi_{1}\right), \Sigma\left(\varphi_{2}\right)\right)$.

A third notion has to be introduced in order to keep the 2-base system of Boolean bits: summand, which denotes every single component or term $\sigma\left(\varphi_{i}\right)$ of the addition $\Sigma\left(\varphi_{i}\right)$. This item helps to define the logical opposition between two arithmetic terms through their characteristic bitstrings, thereby focusing on the essential role of Boolean algebra to connect logic and arithmetic.

Arithmetic oppositions can now be defined with the help of the above three main concepts of maximal value, minimal value, and summand.

For every logical relation $\operatorname{Op}\left(\Sigma\left(\varphi_{1}\right), \Sigma\left(\varphi_{2}\right)\right)$ between integers, we have: 
Contrariety

$\operatorname{Op}\left(\Sigma\left(\varphi_{1}\right), \Sigma\left(\varphi_{2}\right)\right)=\mathrm{CT}\left(\Sigma\left(\varphi_{1}\right), \operatorname{ct}\left(\Sigma\left(\varphi_{1}\right)\right)\right)$ iff

- $\sigma\left(\varphi_{1}\right) \neq 0 \Rightarrow \sigma\left(\varphi_{2}\right)=0$

- $\sigma\left(\varphi_{1}\right)=0 \Rightarrow / \sigma\left(\varphi_{2}\right) \neq 0$

Example: $\mathrm{CT}(p \wedge q, p \wedge \sim q)$

$\Sigma(p \wedge q)=8=8+0+0+0 ; \Sigma(p \wedge \sim q)=0100=0+4+0+0$.

Contradictoriness

$\operatorname{Op}\left(\Sigma\left(\varphi_{1}\right), \Sigma\left(\varphi_{2}\right)\right)=\mathrm{CD}\left(\Sigma\left(\varphi_{1}\right), \operatorname{cd}\left(\Sigma\left(\varphi_{1}\right)\right)\right)$ iff

$-\sigma\left(\varphi_{1}\right) \neq 0 \Rightarrow \sigma\left(\varphi_{2}\right)=0$

- $\sigma\left(\varphi_{1}\right)=0 \Rightarrow \sigma\left(\varphi_{2}\right) \neq 0$

Example: $\mathrm{CD}(p \wedge q, \sim(p \wedge q))$

$\Sigma(p \wedge q)=8=8+0+0+0 ; \Sigma(\sim(p \wedge q))=7=0+4+2+1$.

Subcontrariety

$\operatorname{Op}\left(\Sigma\left(\varphi_{1}\right), \Sigma\left(\varphi_{2}\right)\right)=\operatorname{SCT}\left(\Sigma\left(\varphi_{1}\right), \operatorname{sct}\left(\Sigma\left(\varphi_{1}\right)\right)\right)$ iff

- $\sigma\left(\varphi_{1}\right) \neq 0 \Rightarrow / \sigma\left(\varphi_{2}\right)=0$

- $\sigma\left(\varphi_{1}\right)=0 \Rightarrow \sigma\left(\varphi_{2}\right) \neq 0$

Example: $\operatorname{SCT}(p \vee q, \sim(p \wedge q))$

$\Sigma(p \vee q)=14=8+4+2+0 ; \Sigma(\sim(p \wedge q))=7=0+4+2+1$.

Non-contradictoriness

$\mathrm{Op}\left(\Sigma\left(\varphi_{1}\right), \Sigma\left(\varphi_{2}\right)\right)=\operatorname{NCD}\left(\Sigma\left(\varphi_{1}\right), \operatorname{ncd}\left(\Sigma\left(\varphi_{1}\right)\right)\right)$ iff

- $\sigma\left(\varphi_{1}\right)=0 \Rightarrow / \sigma\left(\varphi_{2}\right) \neq 0$

- $\sigma\left(\varphi_{1}\right) \neq 0 \Rightarrow / \sigma\left(\varphi_{2}\right)=0$

Example: $\mathrm{NCD}(p, q)$

$\Sigma(p \wedge q)=12=8+4+0+0 ; \Sigma(p \vee q)=6=0+4+2+0$.

Subalternation

$\operatorname{Op}\left(\Sigma\left(\varphi_{1}\right), \Sigma\left(\varphi_{2}\right)\right)=\operatorname{SB}\left(\Sigma\left(\varphi_{1}\right), \operatorname{sb}\left(\Sigma\left(\varphi_{1}\right)\right)\right)$ iff

- $\sigma\left(\varphi_{1}\right) \neq 0 \Rightarrow \sigma\left(\varphi_{2}\right) \neq 0$

- $\sigma\left(\varphi_{1}\right)=0 \Rightarrow / \sigma\left(\varphi_{2}\right) \neq 0$

Example: $\mathrm{SB}(p \wedge q, p \vee q)$

$\Sigma(p \wedge q)=8=8+0+0+0 ; \Sigma(p \vee q)=14=8+4+2+0$.

In this way, arithmetic oppositions can make sense thanks to a correspondence with logical values in QAS and their arithmetic properties. As such, the third and final stage of our analogy concerns the link between arithmetic and geometry of oppositions.

\subsection{Analytic geometry of oppositions}

The analogy between arithmetic and geometry is made in the light of what Descartes devised under the heading of analytic geometry. Its present version includes three main components, namely: coordinates, identity, and opposition.

Just as in analytic geometry, expressions like points are to be defined by coordinates in a space. Let $A$ and $B$ be any two points in a 2-dimensional vector space; then $A B \rightarrow=\left(x_{B}-x_{A}\right.$; 
$\left.\mathrm{y}_{\mathrm{B}}-\mathrm{y}_{\mathrm{A}}\right)$. In our arithmetic of oppositions, coordinates are bitstrings and vectors are turned into constants arithmetic functions $\oplus$ between integers.

Example: let \pm for $+2, \Sigma(\varphi)=3$; hence $\pm(\varphi)=3+2=5$.

Furthermore, identity has a precise definition both in analytic geometry and arithmetic.

Let $\mathrm{A}, \mathrm{B}, \mathrm{C}$ be any three points in a vector space. Then the corresponding vectors $\mathrm{AB}^{\rightarrow}=u \rightarrow$ and $\mathrm{BC}^{\rightarrow}=v^{\rightarrow}$ are identical in a given space if and only if they have:

- the same direction

- the same sense

- the same norm

In the structured geometry of oppositions, the first criterion means that any two lines relating vertices are parallel with each other. The second criterion has one logical counterpart: subalternation, where the arrow between vertices indicates the sense of entailment from premise to conclusion. The third criterion cannot be explained in logical terms: a norm is a distance that cannot be rendered in a logical space, and only an arithmetization of oppositions can do this with the help of an operator of differentiation \pm .

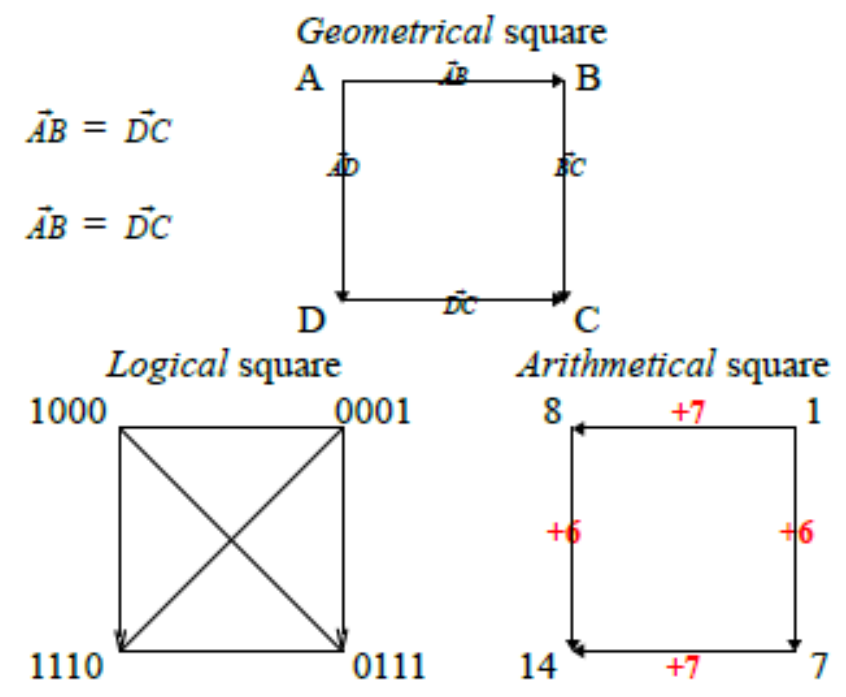

Finally, the idea of opposite vectors is the most important and proceeds as the converse of identity. However, "opposite" is to be made in a particular sense of maximal opposition.

Two vectors $u$ and $v \rightarrow$ are opposed to each other if and only if they have opposite coordinates, such as: $u^{\rightarrow}=-v \overrightarrow{ }$.

If such geometric relations and properties are verified in a logical space of oppositions, modulo its Boolean transcription of logical values in QAS, then the same features of analytic geometry should equally hold in our arithmetic of oppositions. An interesting result can be obtained in this respect, concerning the calculus of oppositions already suggested in [7] but limited by the inner constraints of one-many operators.

Another relevant result for our own purposes is the application of the so-called Chasles' relation in analytic geometry.

For any points $A, B, C$ in an affine space, we have:

$\mathrm{AC}^{\rightarrow}=\mathrm{AB}^{\rightarrow}+\mathrm{BC}^{\rightarrow}$ 
The same properties can be observed in the 2D geometry of oppositions, as illustrated by the following half-squares of opposition.
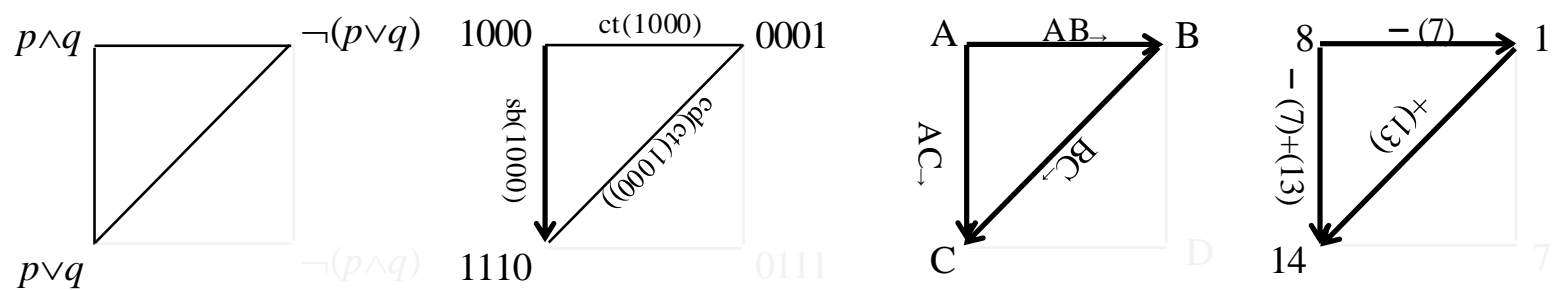

Given the definitions of coordinates and vectors given above, the correspondence of diagrams shows that Chasles' relation applies to 2D geometries only. That it is, to Aristotle's square, but also Blanché's hexagon, Buridan's octagon, and so on. However, a reference to Pellissier's tetraicosahedron also shows that it does not hold for 3D geometries: the identity criteria of vectors are lost once the third dimension of depth is introduced between vertices. For this reason, we limit our correspondence result to 2D geometries for the same reason Chasles' relation holds for affine spaces only.

Thanks to this arithmetization of logical oppositions, it is also possible to identify any logical relation between expressions. The one-many operators of logical oppositions made this impossible, whereas a calculus of single values helps to define any sort of relation on the basis of its relata. So, the correspondence also confirms the plurality of logical negations as expounded in [7] from a purely Boolean perspective of logical negations.

Echoing the previous picture of oppositions as a set of partial identities and differences, it is easily seen that not every double negation amounts to mere affirmation in a logical calculus of oppositions: far from that, given the occurrence of double mixed negations like, e.g., $\operatorname{ct}\left(\operatorname{cd}\left(\varphi_{i}\right)\right)$. Thus, for any opposition-forming operators op $x$ and $\mathrm{op}_{y}$ :

$$
\mathrm{op}_{x} \mathrm{op}_{y}(\varphi)=\varphi \text { iff } x=y=\mathrm{cd}
$$

This does not hold for the other logical oppositions such as contrariety. A contrary of a contrary of $\varphi$ can be another contrary of $\varphi$, and the same holds for every one-many mapping whereas contradictoriness proceeds as a genuine function, i.e. a one-one operator.

More importantly, arithmetic helps to overcome the obstacle of one-many operators by specifying the Boolean calculus of opposites: although there is not only one contrary, for example, an arithmetic calculus helps to operate between single identified opposites from particular relata.

To give an example of such a calculus, let us take any binary sentence of Pellissier's tetraicosahedron as a starting point; then let us check the final value of its opposite through a finite sequence of operations between the 16 integers from 0 to 15 .

Let $\mathbf{A}(p \wedge q)=1000=\Sigma(p \wedge q)=8$. Then:

$8-5=3=0+0+2+1=\mathbf{A}(0011)=\Sigma(\neg p), 0011 \subseteq \operatorname{ct}(1000)$; therefore, $\mathrm{Op}(8,3)=\mathrm{CT}(p \wedge q, \neg q)$.

$3+11=14=8+4+2+0=\mathbf{A}(1110)=\Sigma(p \vee q), 1110 \subseteq \operatorname{sct}(0011)$; therefore $\mathrm{Op}(3,14)=$ $\operatorname{SCT}(p \wedge q, \neg q)$.

$14-2=12=8+4+0+0=\mathbf{A}(1100)=\Sigma(p), 1100 \subseteq \operatorname{sp}(1110)$; therefore, Op $(14,12)=\operatorname{SP}(p \vee q, p)$. $8-5+11-2=12=8+4+0+0=\mathbf{A}(1100)=\Sigma(p), 1100 \subseteq \operatorname{sb}(1000)$; therefore, $\operatorname{Op}(8,12)=$ $\mathrm{SB}(p \wedge q, p)$. 
To summarize:

- $p$ is a subaltern of $p \wedge q: \operatorname{sb}(p \wedge q) \supseteq p$.

- $p$ is a contrary of a subcontrary of a superaltern of $p \wedge q$ : $\operatorname{ct}(\operatorname{set}(\operatorname{sp}(p \wedge q))) \supseteq p$.

It is worthwhile recalling that the above operations are not equations $x=\operatorname{op}(y)$ between single values but, rather, inclusions $x \subseteq \mathrm{op}(x)$ between singletons and sets. This is the case, because there is not only one subaltern to binary conjunction, and the above sequence is just one possible way to move from an expression to one of its subalterns through arithmetic extensions.

\section{Conclusion}

In this paper, I have proposed an arithmetization of logical oppositions, based on a structural identity between three domains: logic, geometry, and arithmetic. Moving from one domain to another one through the central device of Boolean algebra is possible by assuming that any operation of a given domain finds its counterpart in the other.

This correspondence result can be summarized in the following table:

\begin{tabular}{|c|c|c|c|}
\hline & Logic & Geometry & Arithmetic \\
\hline$(1)$ & $\varphi_{1}$ & A & $x \in \mathrm{N}^{+}$ \\
\hline$(2)$ & $\varphi_{2} \subseteq \mathrm{op}\left(\varphi_{1}\right)$ & $\mathrm{B}$ & $y \in \mathrm{N}^{+}$ \\
\hline$(3)$ & $\mathrm{Op}\left(\varphi_{1}, \varphi_{2}\right)$ & {$[\mathrm{AB}]$} & $\oplus(x, y)$ \\
\hline$(4)$ & $\mathrm{op}$ & $u^{\rightarrow}=\mathrm{AB} \rightarrow$ & $\pm(x)=y$ \\
\hline
\end{tabular}

According to this table:

(1) expressions are like different points in geometry and different integers in arithmetic;

(2) opposites are like differentiated points in geometry and differentiated integers in arithmetic;

(3) opposition is like a segment in geometry and a numerical difference in arithmetic;

(4) opposition-forming operators are like norms in analytic geometry and constant operations of addition or subtraction in arithmetic.

The whole process may be seen as a legacy of Leibniz's achievements in logic. That is, despite its limited results, the algebraic logic of oppositions is an instantiation of what Leibniz took as a lingua characteristic. Each relation of opposition is definite in a finite set of expressions, and not every element of a given language can be compared to any other in terms of oppositions. Likewise, the proposed arithmetic may be understood in terms of a calculus ratiocinator, by computing the logical relation of expression through their arithmetic values.

A number of further problems have not been addressed in the present paper and should be developed in later works. For instance, how to construct a general algebraic logic for any sorts of sentences beyond the sole binary sentences $p \circ q$ and its 16 elements? This will depend upon the capacity of QAS to characterize every meaningful expression in the form of a bitstring. This has been achieved with modal expressions [8-10], but only under the proviso that their sense be given by a different set of questionings.

Furthermore, the above arithmetic calculus relies upon a decimal coding of Boolean values. The underlying game of yes-no answers thereby assumed a bivalent algebra $\{1,0\}$. What of the meaning of expressions in non-Boolean algebras? If bivalence is rendered by yes-no answers 1-0 and results in a 2-base number system, then many-valuedness amounts to a 
higher set of $1, \ldots, k$ answers in QAS (where $k>2$ ) and should require a corresponding $k$-base system.

\section{References}

1. Béziau, J.-Y. New light on the square of oppositions and its nameless corner. Log. Investig. 10, 218-233 (2003)

2. Blanché, R. Sur l'opposition des concepts, Theoria 19, 89-130 (1953)

3. Blanché, R. Sur la structuration du tableau des connectifs interpropositionnels binaires. The J. of Symb. Log. 22, 178 (1957)

4. Czeżowski, T. On certain peculiarities of singular propositions. Mind 64, 392-395 (1955)

5. Moretti, A. The Geometry of Logical Opposition. PhD Thesis, University of Neuchatel, 2009

6. Pellissier, R. 'Setting' n-opposition. Logica Universalis 2, 235-263 (2008)

7. Schang, F. Oppositions and opposites. In: Around and Beyond the Square of Opposition, Béziau, J.Y. \& Jacquette, D. (eds.), Birkhäuser/Springer Basel, 147-173 (2012)

8. Schang, F. Logic in Opposition. Studia Humana 2(3), 31-45 (2013)

9. Schang, F. No, No, and No. Submitted draft (201X)

10. Smessaert, H. On the 3D-visualisation of logical relations. Logica Universalis 3: 303-332

11. Smessaert, H. \& Demey, L. Logical Geometries and Information in the Square of Oppositions. J. of Log., Lang. and Inf. 23(4), 527-565 (2014)

F. Schang $(\bowtie)$

National Research University Higher School of Economics, Moscow, Russia

e-mail: schangfabien@gmail.com

email: fschang@ hse.ru

\section{Appendix: A constructive geometry of logical relations}

In the first section of the paper, a historical reference has been made to Shao Wong's ordering of the 64 hexagrams. Its striking feature is that it also respects the central symmetry of contradictory oppositions between the Boolean bitstrings - and their corresponding blue integers, here below, as is the case in all contemporary gatherings of logical geometry.

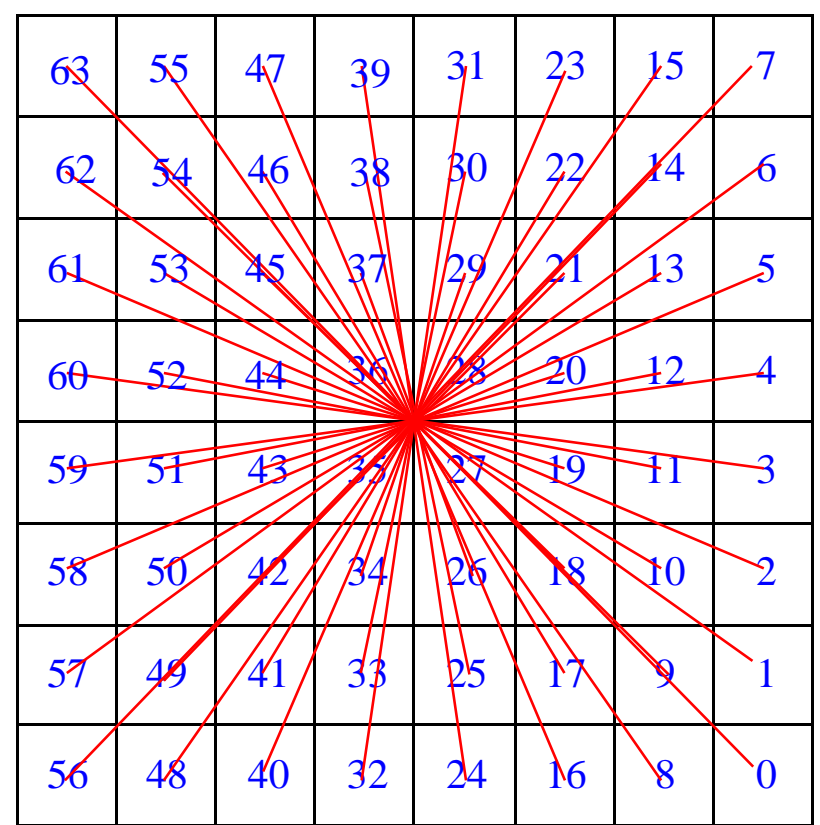


We propose in the following a similar constructive representation of logical oppositions: all are decreasing quadrangles, of length $L$ and width $l$. Each progression of a given $2^{n}$ quadrangle consists in duplicating it either horizontally (from left to right) when $n$ is odd, or vertically (from top to bottom) when $n$ is even. The resulting figure is either a rectangle, such that $L=2 l$ whenever $n$ is odd, or a square, such that $L=l$ whenever $n$ is even. Each quadrangle is a complete set of bitstrings from the minimal value 0 to the maximal value $2^{n}-$ 1 , and the new ordering also preserves the properties of vectors (except Chasles' relation).

$$
2^{1}=2
$$

\begin{tabular}{|l|l|}
\hline 1 & 0 \\
\hline 1 & 0 \\
\hline
\end{tabular}

$$
2^{4}=16
$$

\begin{tabular}{|c|c|c|c|}
\hline 15 & 13 & 7 & 5 \\
1111 & 1101 & $0 / 11$ & 0101 \\
\hline 14 & 2 & 6 & 4 \\
1110 & 1100 & 4110 & 0100 \\
\hline 11 & 9 & 3 & 1 \\
1011 & 100 & 001 & 0001 \\
\hline 10 & $\$$ & 2 & 0 \\
1010 & 1000 & 0010 & 0000 \\
\hline
\end{tabular}

$$
2^{2}=4
$$

\begin{tabular}{|c|c|}
\hline 3 & 1 \\
11 & 01 \\
\hline 2 & 0 \\
10 & 00 \\
\hline
\end{tabular}

$$
2^{3}=8
$$

\begin{tabular}{|c|c|c|c|c|c|c|c|}
\hline 11111 & $\begin{array}{r}30 \\
+1110 \\
\end{array}$ & $\begin{array}{r}23 \\
10111 \\
\end{array}$ & $\begin{array}{c}22 \\
10 \perp 10 \\
\end{array}$ & $\begin{array}{c}15 \\
0 / 111\end{array}$ & 14 & $\frac{7}{00141}$ & $\begin{array}{c}6 \\
00110\end{array}$ \\
\hline 29 & 28 & & & & 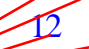 & 5 & -4 \\
\hline 11101 & 11100 & 10101 & Dat & & ण1100 & 00101 & 00100 \\
\hline 27 & 26 & & & & & 3 & -2 \\
\hline 11011 & 11010 & 10911 & 1000 & d1011 & 01010 & 00011 & 00010 \\
\hline $\begin{array}{c}25 \\
11001\end{array}$ & & $\begin{array}{c}17 \\
10001\end{array}$ & $\begin{array}{c}16 \\
10000\end{array}$ & $\begin{array}{c}9 \\
01001\end{array}$ & $\begin{array}{c}8 \\
01000\end{array}$ & $\begin{array}{c}1 \\
00001\end{array}$ & $\begin{array}{c}0 \\
00000\end{array}$ \\
\hline
\end{tabular}

\begin{tabular}{|c|c|c|c|}
\hline 7 & 6 & 3 & 2 \\
111 & 119 & 211 & 010 \\
\hline 5 & 4 & 1 & 0 \\
\hline 101 & 100 & 001 & 000 \\
\hline
\end{tabular}

$2^{5}=32$

$2^{6}=64$

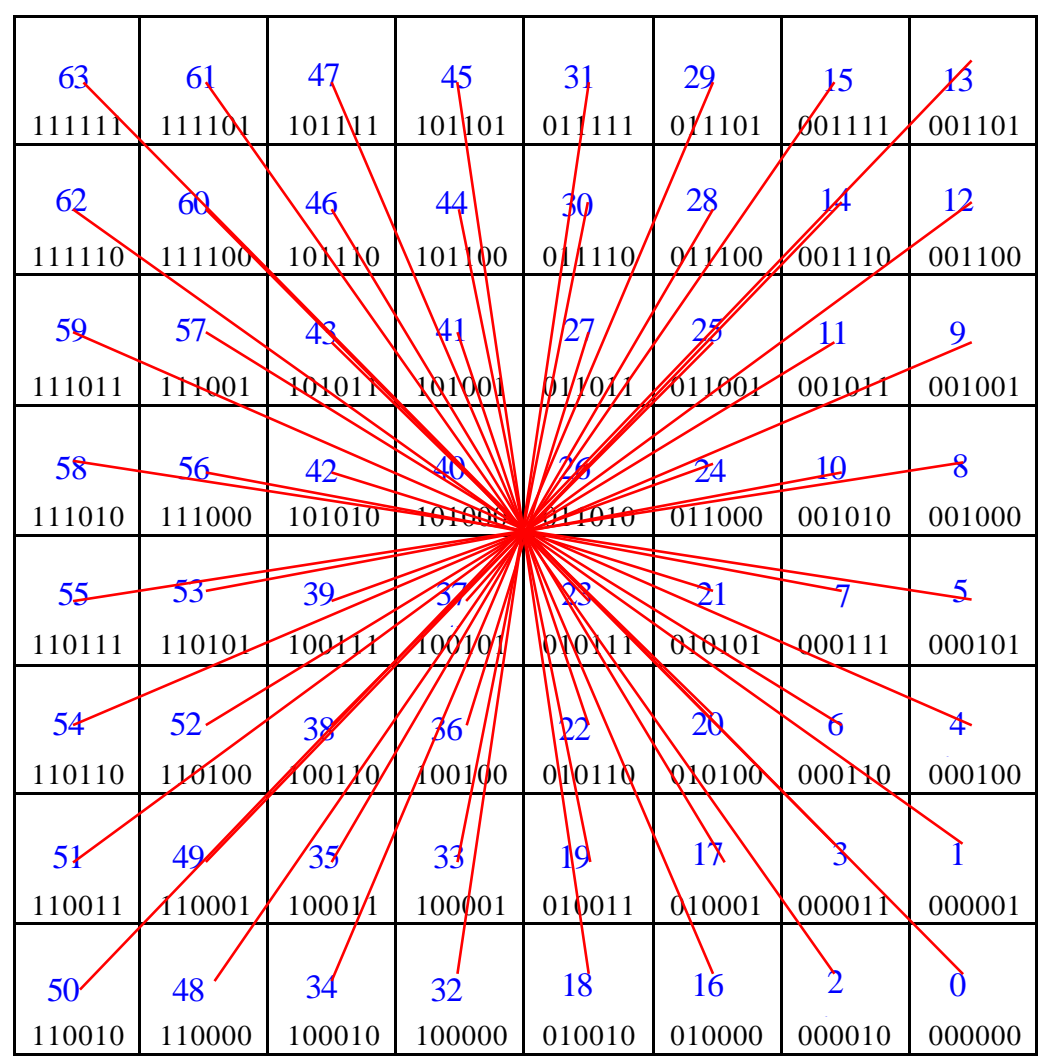

